\title{
QUADRATIC APPROXIMATION AND ITS APPLICATION TO ACCELERATION OF CONVERGENCE
}

\author{
S.L. LOI
}

This thesis is a study of quadratic approximation and its application to the acceleration of slowly converging sequences arising, in particular, from the numerical integration of an (improper) integral with singularity.

It commences by considering the convergence of the quadrature approximants to the integral with singularity. The location of singularity affects the rate of convergence when the process of ignoring the singularity is used. Numerical results show that most of the quadratures can cater for the integral with a singularity located at the end points of the interval (or subintervals) of integration.

Then the rate of convergence is considered. A number of accelerators such as the Romberg method, Aitken $\Delta^{2}$ process and the $\varepsilon$-algorithm are compared. The $\varepsilon$-algorithm is not as good as the modified Romberg method, but better than the unmodified Romberg and Aitken $\Delta^{2}$ process.

Since the $\varepsilon$-algorithm is based on the use of the Pade approximation, a general algorithm for the calculation of rational approximations is developed.

The quadratic approximation, which is an extension of Pade approximation, is then studied. The general forms of expressing this approximation by the polynomial coefficients are given. A recursive algorithm for the construction of the approximation is derived. This algorithm is based on the Muhlbach-Neville-Aitken algorithm which can be

Received 8 November 1982. Thesis submitted to University of Canterbury, July 1982. Degree approved, October 1982. Supervisor: Dr A. W. McInnes. 
applied to the rational interpolation and Pade approximation. This approximation can be applied to speed up the convergence of a converging sequence and can be extended to the interpolation. With the general recursive algorithm, all possible quadratic approximants and interpolants can be constructed.

The use of the quadratic approximation for linearly convergent sequences, monotonic and alternating series is illustrated. Numerical results show that the convergence of a suitable quadratic approximant is faster than Pade approximant if convergence of the Pade approximant is not fast. Some properties of quadratic approximations are derived.

Department of Mathematics, National University of Singapore, Kent Ridge, Singapore 0511 . 